\title{
The surgical anatomy for multiple-electrode extracochlear implant operations
}

\author{
Burkhard K-H. G. Franz and Graeme M. Clark (Melbourne, Australia)
}

\begin{abstract}
Direct access to the whole length of the cochlear turns via endaural middle ear approach for the placement of extracochlear electrodes is severely restricted. Approximately $10 \mathrm{~mm}$. of the cochlear turns are accessible, being less than a third of their length. The middle cranial fossa, the facial nerve, the internal carotid artery and the temporomandibular joint restrict the acccss. A further restriction is caused by the position of the cochlea and the direction of its axis. The anterior part of the cochlea lies anterior to the tympanic membrane and medial to the temporomandibular joint, thus limiting an endaural approach to a posterolateral direction. Despite this limitation small sections of the basal, middle and apical turns of the cochlea can be reached.
\end{abstract}

\section{Introduction}

The intracochlear multiple-electrode stimulation of residual nerve fibres in the totally deaf patient has resulted in speech perception results that are generally better than for single-electrode extracochlear or single intracochlear stimulation (Gantz et al.,1987). Not every patient, however, is suitable for an intracochlear implant. Chronic middle ear infections, labyrinthitis ossifcans and possibly malformations of the inner ear render the patient unsuitable. In these cases the extracochlear multiple-electrode implant may be an alternative. It has a potential advantage over extracochlear single-electrode stimulation in that some place pitch perception may be possible.

Descriptions of the surgical anatomy for multipleelectrode extracochlear implantation (Banfai et al.,1980, 1984 ; Kubik, 1984) have indicated that a large part of the cochlear turns are projected to the medial wall of the middle ear, and therefore accessible to the placement of overlying electrodes. Previous studies in our department, however, concluded that the overall length of the cochlear turns accessible in the middle ear is approximately $8 \mathrm{~mm}$. (Clark, 1975). and this would enable the placement of electrodes over only a small part of the cochlear turns. This study has been undertaken to provide more data and help resolve this situation.

\section{Method}

Six adult human temporal bones were used in this study. Four bones were dissected to study the accessibility of the cochlea via an endaural middle ear approach. The cochlear turns projecting to the medial wall of the middle ear were skeletonized as extensively as possible leaving the middle cranial fossa, the facial nerve, the internal carotid artery and the the temporomandibular joint intact. The lengths of the exposed turns were measured along their outer periphery. The cochlear turns projecting to the middle cranial fossa were also skeletonized leaving the roof of the middle ear, the facial nerve, the greater superficial petrosal nerve and the internal carotid artery intact. The lengths of these turns were measured along their outer periphery. The cochlear turns adjacent to the internal carotid artery were also exposed and their lengths measured. The position of the cochlea and its axis was noted and compared to the position of the remaining structures of the labyrinth and the middle ear.

In addition, the cochleas of two bones were skeletonized specifically to determine the number and best possible locations for electrodes $1 \mathrm{~mm}$. in diameter that could overlie the cochlea to provide place pitch perception.

The steps of all procedures were photographed, and documented by diagrams. Dissections were carried out under an operating microscope.

\section{Results}

The dissections showed that a $1 \mathrm{~mm}$. electrode can be placed over the apical region of the cochlea, found in a triangle formed by the internal carotid artery, the tensor tympani muscle and the roof of the middle ear (Fig. 1). Two electrodes can be placed over the middle turn of the cochlea, found between the anterior edge of the oval window and the internal carotid artery (Fig.l). One or two electrodes can be placed on the promontory over the basal turn. An electrode can also be placed into the round window niche (Fig.l). Access to the anterior half of the cochlea is restricted as it lies medial to the tem- 


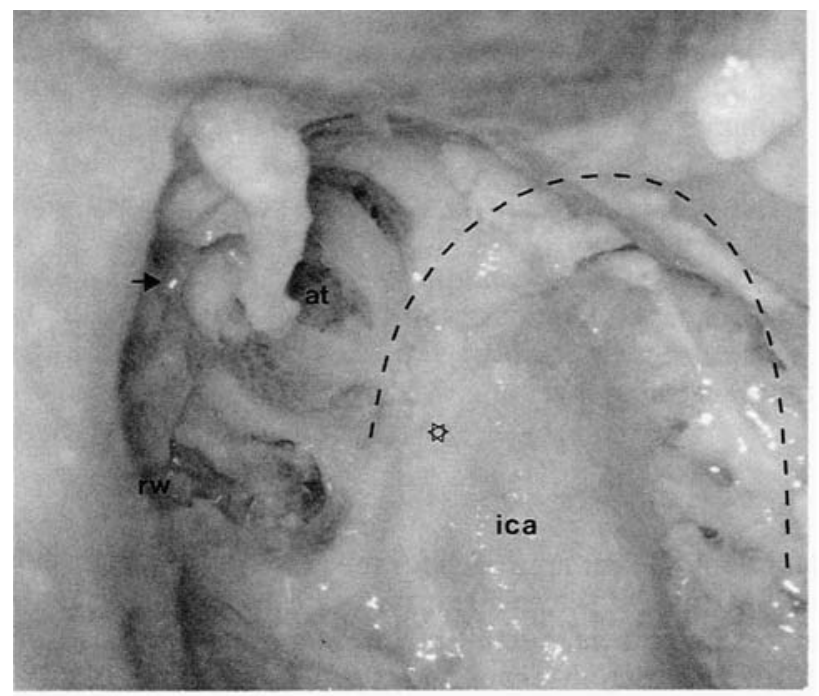

Fig. 1

Photograph demonstrating the cochlear turns projected to the medial wall of the middle ear. View through the external ear canal. The temporomandibular joint is removed (dotted). The position of the middle turn is found on a line connecting the anterior edge of the oval window (arrow) with the internal carotid artery (asterix). ica: internal carotid artery, rw: round window, at: apical turn.

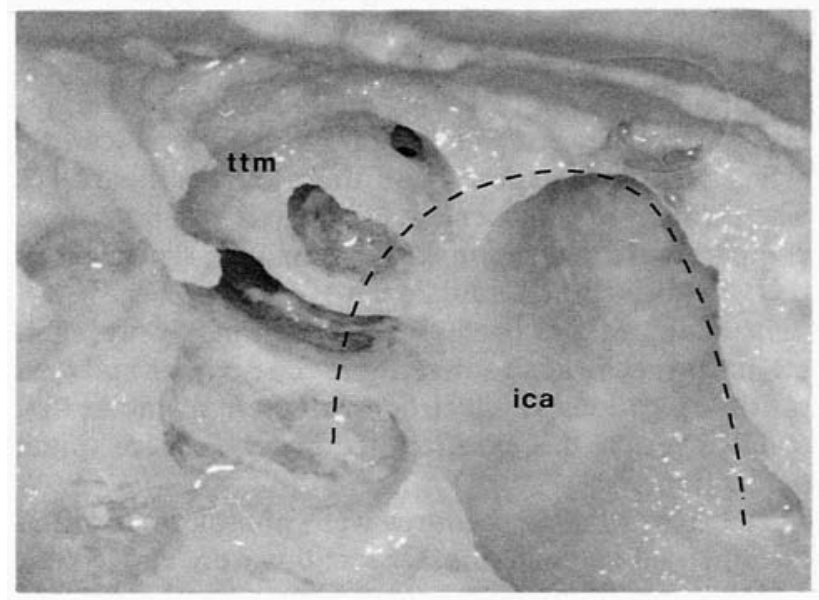

FIG. 2

Photograph demonstrating the relation of the cochlear turns to the internal carotid artery and temporomandibular joint (dotted). View from antero-lateral direction. ica: bed of internal carotid artery, ttm: bed of resected tensor tympani muscle.

TABLE I

LENGTH IN MM OF COCHLEAR TURNS PROJECTED TO THE MEDIAL WALL OF THE MIDDLE EAR

\begin{tabular}{lccccc}
\hline & \multicolumn{3}{c}{ Temporal bone specimen } & \multirow{2}{*}{ Average } \\
\cline { 2 - 5 } & 1 & 2 & 3 & 4 & \\
\hline apical turn & 2.0 & 1.0 & 2.0 & 1.5 & 1.6 \\
middle turn & 3.8 & 2.8 & 3.5 & 4.0 & 3.5 \\
basal turn & 4.5 & 5.0 & 4.5 & 5.0 & 4.8 \\
\hline
\end{tabular}

TABLE II

LENGTH IN MM OF COCHLEAR TURNS PROJECTED TO THE INTERNAL CAROTID ARTERY

\begin{tabular}{lccccc}
\hline & \multicolumn{4}{c}{ Temporal bone specimen } & Average \\
\cline { 2 - 5 } & 1 & 2 & 3 & 4 & \\
\hline middle turn & 1.8 & 2.5 & 2.0 & 1.8 & 2.0 \\
basal turn & 3.5 & 3.5 & 4.0 & 3.8 & 3.7 \\
\hline
\end{tabular}

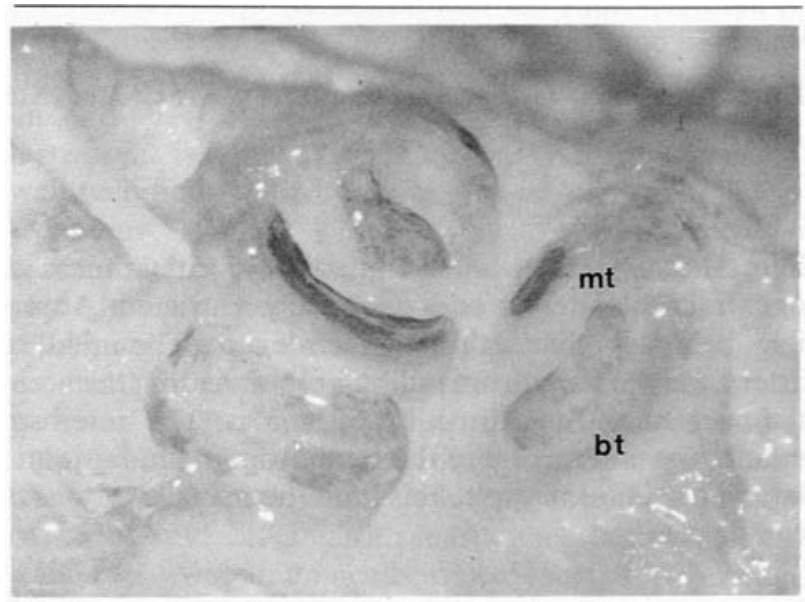

Fig. 3

Photograph demonstrating the relation of the cochlear turns and the internal carotid artery; view from antero-lateral. The temporomandibular joint and the internal carotid artery removed, sections of the basal turn and middle turn previously covered by the internal carotid artery exposed. bt: section of basal turn, mt: section of middle turn.

TABLE III

LENGTH IN MM OF COCHLEAR TURNS PROJECTED TO THE MIDDLE CRANIAL FOSSA

\begin{tabular}{lccccc}
\hline & \multicolumn{4}{c}{ Temporal bone specimen } & \\
\cline { 2 - 5 } & 1 & 2 & 3 & 4 & Average \\
\hline apical turn & 1.0 & 1.3 & 1.5 & 1.0 & 1.2 \\
middle turn & 1.8 & 2.0 & 2.5 & 2.3 & 2.2 \\
basal turn & 4.0 & 3.8 & 4.0 & 3.9 & 4.0 \\
\hline
\end{tabular}

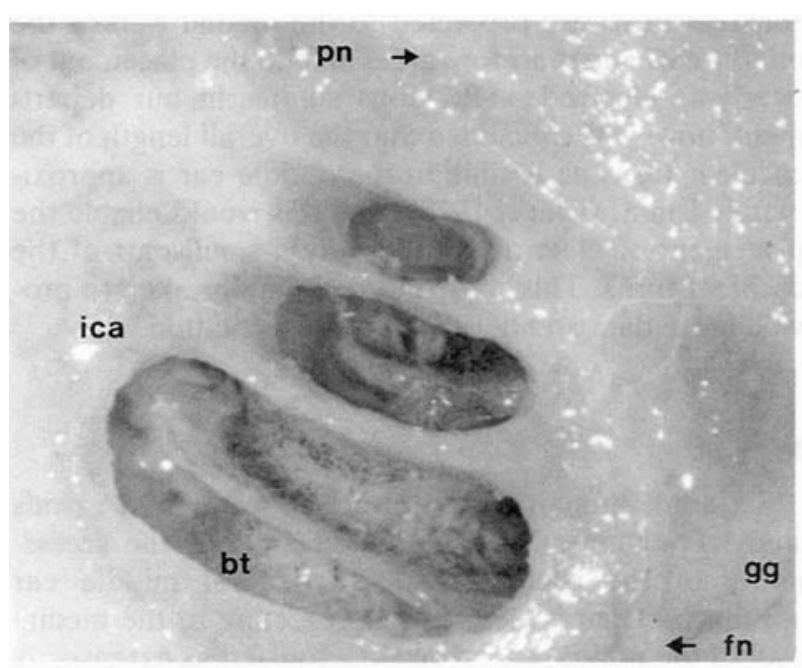

Fig. 4

Photograph demonstrating the cochlear turns projected to the middle cranial fossa. pn: greater petrosal nerve, gg: geniculate ganglion, fn: facial nerve, ica: internal carotid artery, bt: upper section of basal turn. 
poromandibular joint (Fig.2). The approach to this must be from a postero-lateral direction.

The modiolus of the cochlea forms an angle of 90 degrees with the axis of the petrous bone. The modiolus of the cochlea is also directed anteriorly at approximately 45 degrees to the coronal plane and superiorly at approximately 30 degrees to the horizontal plane. Although the modiolus is directed anteriorly, superiorly and laterally, the middle and apical turns of the cochlea are not readily accessible because of the prominence of the basal turn, and the thickness of the bone overlying the apical and middle turns.

The anatomical dissections also showed that the first $4.5-5 \mathrm{~mm}$. of the basal turn, the first $2.8-4 \mathrm{~mm}$. of the middle turn and $1.5-2 \mathrm{~mm}$. of the apical region of the cochlea project to the medial wall of the middle ear (Table I) . 3.5-4 mm. of a more distal section of the basal turn and $1.8-2.5 \mathrm{~mm}$ of the more distal section of the middle turn are covered by the internal carotid artery (Table II) (Figs.2 and 3). The remaining $3.8 \mathrm{~mm}$. of the basal turn, and $1.8-2.5 \mathrm{~mm}$. of the middle turn and $1-1.5 \mathrm{~mm}$. of the upper turn are projected to the middle cranial fossa (Table III) (Fig.4). It is also of interest to note that the tensor tympani muscle crosses the apical region of the cochlea, and this can be exposed more generously when the muscle is dissected.

In summary, when the cochlea is skeletonized from the middle ear $9.9 \mathrm{~mm}$. of the cochlear turns are exposed. When the cochlea is skeletonized from the middle cranial fossa $7.4 \mathrm{~mm}$. of the cochlear turns are exposed. Finally the internal carotid artery covers $5.7 \mathrm{~mm}$. of the cochlear turns.

\section{Discussion}

The concept of a multichannel cochlear implant system is supported by the finding that a better understanding of speech is achieved when nerve fibres are stimulated on the basis of place pitch and rate pitch (timing) codes. Best results are also obtained when electrodes are placed as close as possible to nerve fibre groups that can be stimulated individually (Black et al., 1983).

Surgical access to individual nerve fibre groups and to different areas of the cochlear turns for the placement of extracochlear electrodes were described (Banfai et al., 1980, 1984; Kubik, 1984). Their results indicated that more than half the length of the cochlear turns can be reached via the middle ear.

The cochlear turns have an average length ranging from 31-33 mm. (Schuknecht, 1974). Previous measurements of the length of the cochlear turns that are projected to the medial wall of the middle ear gave an average value of $8 \mathrm{~mm}$. (Clark, 1975). These measurements were confirmed in this study and thus indicate that a small proportion of the length of the cochlear turns is suitable for the placement of overlying extracochlear electrodes.

The length of the cochlear turns projecting to the medial wall of the middle ear, to the internal carotid artery and to the medial cranial fossa totalled in our study $23 \mathrm{~mm}$. This is less than the estimated total length of the cochlear turns. The reason for the discrepancy is due to the bony bridges which separated the three dissected sectors of the cochlea, and which were not included in the measurements nor removed completely in order not to disturb the anatomical boundaries. The lengths of the cochlear turns projecting to the medial wall of the middle ear, however, were measured after having been exposed as far as it was possible via an endaural middle ear approach.

Using an endaural middle ear approach there appear to be a number of reasons responsible for a restricted access to the cochlear turns. The temporomandibular joint overlies the cochlea anteriorly. The axis of the cochlea is in an antero-latero-superior direction. The basal turn is prominent and the bone is thick over the apical and middle turns. The internal carotid artery is in close contact with the cochlea and prevents access to sections of the basal and middle turns. The horizontal portion of the facial nerve lies over the cochlea where the basal and middle turns pass from beneath the middle cranial fossa to the medial wall of the middle ear (Fig.4). The greater superficial petrosal nerve and the geniculate ganglion prevent access to distal sections of the basal and middle turns, which lie under the middle cranial fossa. The tensor tympani muscle does not prevent access to the apical region of the cochlea. When this muscle is dissected the apical region can be exposed more generously. A further difficulty in siting electrodes over the apical and middle turns is that each cochlear turn is overlapped or embraced by the next turn in an apical to basal direction. This results in only the scala vestibuli of the apical and middle turns being exposed.

In order to take advantage of the tonotopic organisation of the cochlea with an extracochlear device it is necessary to place the electrodes at different points around the cochlear turns and as close as possible to individual nerve fibre groups. The limited access to only a small sector of the cochlea must restrict the number of electrodes that can be placed along the cochlear turns. Assuming the size of the electrode to be $1 \mathrm{~mm}$. in diameter, only five electrodes can be placed comfortably along the cochlear turns. This is a much smaller number of electrodes than is currently used for the multi-electrode extracochlear implant (Banfai et al., 1986; Pulec, 1986).

As the cochlear turns lie anteriorly under the medial wall of the middle ear, a better surgical approach is achieved by making a postero-superiorly based tympanomeatal flap. The handle of the malleus thus lies posteriorly and can be left in-situ. The incision in the endomeatal skin of the anterior and inferior canal walls is extended posteriorly sufficiently far to provide adequate exposure of the round window niche. To improve the access it might also be necessary to reduce the anterior recess of the external ear canal until a thin bony plate remains over the temporomandibular joint for protec. tion. The majority of the middle ear structures can be left intact. The preservation of the middle ear structures can be important especially in cases were implantation is carried out in patients with some residual hearing.

\section{Conclusion}

Less than a third of the cochlear length is accessible from an endaural middle ear approach for the placement of individual extracochlear electrodes. Despite this limitation the basal turn, the middle turn and the apical turn can be reached. 


\section{References}

Banfai, P., Hartmann, G., Kubik, S., and Wustrow, F. (1980) Projection of the spiral cochlear canal on the medial wall of the tympanic cavity with regard to the cochlear implant. Scandinavian Audiology Supplement, 11: 157-162.

Banfai, P., Kubik, S. and Hartmann, G. (1984) Our extrascalar operating method of cochlear implantation. Acta Otolaryngologica, Supplement, 411: 9-12.

Banfai, P., Karczag, A., Kubik, S., Luers, P. and Suerth, W. (1986) Sixteen channel extracochlear electrode stimulation of the auditory nerve with programmable speech processor. International Conference on: Controversies in Otology and Otoneurosurgery, Alghero.

Black, R.C., Clark, G.M., Tong, Y.C., and Patrick J.F. (1983) Current distribution in cochlear stimulation. Annals of the New York Academy of Sciences, 405: 137-145.

Clark, G.M. (1975) A surgical approach for the cochlear implant: an anatomical study. Journal of Laryngology and Otology, 89: 9-15.
Gantz, B.J., Mccabe, B.F., Tyler, R.S. and Preece J.P. (1987) Evaluation of four cochlear implant designs. Annals of Otology, Rhinology and laryngology, Supplement 128: 145-147.

Kubik, S. (1984) Anatomy of possible approaches for cochlear implant. Advances in Audiology, 2: 108-112.

Pulec, J. (1986) Advantages of the transcutaneous eight channel extracochlear implants. International Conference on: Controversies in Otology and Otoneurosurgery, Alghero.

Schuknecht, H. (1974) Pathology of the ear. Harvard University press.

Address for correspondence:

Burkhard K-H. G. Franz MD,

Department of Otolaryngology,

University of Melbourne,

The Royal Victorian Eye and Ear Hospital,

32 Gisborne St., East Melbourne, Australia. 


\section{University Library}

\section{- M M I E E R VA A gateway to Melbourne's research publications}

Minerva Access is the Institutional Repository of The University of Melbourne

Author/s:

Franz, Burkhard K-H. G.;Clark, Graeme M.

Title:

The surgical anatomy for multiple-electrode extracochlear implant operations

Date:

1988

Citation:

Franz, B. K. G., \& Clark, G. M. (1988). The surgical anatomy for multiple-electrode

extracochlear implant operations. Journal of Laryngology and Otology, 102(8), 685-688.

Persistent Link:

http://hdl.handle.net/11343/27267 